\title{
A COMPREHENSIVE DFT API FOR SCIENTIFIC COMPUTING
}

\author{
Ping Tak Peter Tang \\ Intel Corporation \\ Santa Clara, CA, USA
}

\begin{abstract}
The Fast Fourier Transform (FFT) algorithm that calculates the Discrete Fourier Transform (DFT) is one of the major breakthrough in scientific computing and is now an indispensable tool in a vast number of fields. Unfortunately, software that provide fast computation of DFT via FFT differ vastly in functionality as well as uniformity. A widely accepted Applications Programmer Interface (API) for DFT would advance the field of scientific computing significantly. In this paper, we formulate an API for DFT computation that encompasses all the functionality that are offered by a number of popular packages combined, allows easy porting from existing codes, and exhibits a systematic naming convention with relatively short calling sequences.
\end{abstract}

Keywords: API, FFT, DFT, scientific computing.

\section{INTRODUCTION}

The Fast Fourier Transform (FFT) algorithm that calculates the Discrete Fourier Transform (DFT) is one of the major breakthrough in scientific computing and is now an indispensable tool in a vast number of fields. Unfortunately, software that provide fast computation of DFT via FFT differ vastly in functionality as well as uniformity. A widely accepted Applications Programmer Interface (API) for DFT would advance the field of scientific computing in several major aspects. First, and most obvious, is that applications programmers can much easily port their codes to different platforms. Second, a standard API would further encourage vendors to produce implementations that are highly optimized. Third, a common API for fundamental computation tasks such as the DFT will lead to advances in computational methods and software that rely on DFT as a building block.

Despite the tremendous benefit of a common API, that it does not exist indicates the amount of obstacles preventing its formulation and 
adoption. The diverse functionality of existing software packages demand that a common API be somewhat inclusive in the capability that it offers. The non-uniformity of existing software packages demand that the common API be natural and amenable to easy porting to it. Finally, the common API must exhibit certain superiority over existing ones to motivate conversion effort of legacy codes.

\section{API OF POPULAR SOFTWARE PACKAGES}

We offer a brief survey of some popular packages: two from the public domain, two from hardware vendors, and two from mathematical library vendors. The software packages and libraries surveyed are, in alphabetical order, Cray libsci, FFTPACK, FFTW, IBM ESSL, IMSL, and NAG. We discuss our findings in relation to functionality and portability.

The first significant differences in functionality is that of the data length $N$ that is supported. ESSL only accepts $N$ that are factorizable into primes no larger than 11 , as well as other minor conditions. While all other packages have some routines that support general $N$, most resort to some order $N^{2}$ algorithm when $N$ is "inconveninet," for example, that of a large prime. Only Cray libsci on Cray's MPP platform (such as the T3E) and FFTW implemented order $N \log N$ algorithm for this situation. The second differences in functionality is that of handling strided data and multiple transforms via one single call. Only ESSL and FFTW support this. The third major differences in functionality is that of performing transform without the use of auxiliary storage. Only NAG supports this.

By portability we mean the ease of an application that uses one library to switch to another library. The paramount problem is that of data representation. The simple case of complex-valued input data illustrates the problem. While most packages in this case expect the input be stored in an array of complex type, NAG requires that the real part and imaginary part of the data be stored separately in two real arrays. Porting from one library to another is difficult without an explict copy that wastes both execution time and memory. The crux of the data representation problem is that in DFT computations, more than one representation are possible that can exploit the special properties that are present in the input or output domain (e.g. real valued sequence, conjugate even sequence). Unfortunately, these representations are not easily portable. Since all packages adopt only one representation for each data domain, major portability problem exist whenever these representations differ. The second portability problem relates to auxiliary 
storage area. The packages differ in their need for explicit reference to these areas, or the requirement of their sizes. The third problem is that of placement of results. Some packages supports by default an "in-place" computation where the result overwrites the input; while others supports "out-of-place" and, in the case of Fortran, expect the user to violate the non-aliasing requirement to obtain an "in-place" functionality.

\section{OBJECTIVES AND DESIGN OF PROPOSED API}

Our vision is that, for DFT computation, the scientific and engineering community would eventually be able to rely on a common interface for which most platforms would provide highly optimized implementations. Our objectives here are to provide a set of APIs that (1) supports a set of functionality that is a superset of the combined functionality of the packages surveyed and allows transition to the new API to be straightforward; (2) exhibits simpleness in the calling sequences; and (3) offers clarity in the naming convention and organization.

\subsection{DEAFAULT OPTIONS AND INDEPENDENT UPDATE}

We accommodate the seemingly contradictory objectives of (1) and (2) by a different style. We illustrate this style by the in-place computation of a single-precision, complex, one-dimensional, forward transform of length 32 .

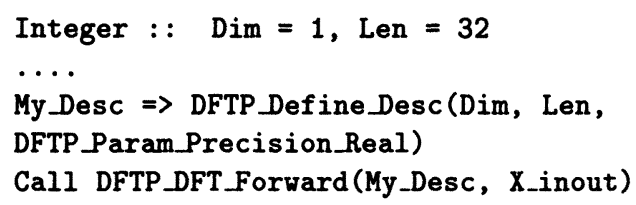

Suppose we would have sixteen transforms of length 32 and that the data is stored contiguously, then prior to the call to DFTP_DFT_Forward, we issue

Call DFTP_Change_NumTransforms (My_Desc, 16)

Briefly speaking, we organize a vast variety of functionality into different options, each with a default. Instead of using multiple parameters in the computation to set choices for each of the many options, we issue a call only to a relevant option changing routine only when the default choice is inappropriate. (The example above suggests that the number of transformations is an option whose default is 1.) 


\subsection{EXPLICIT RECOGNITION OF DOMAIN AND REPRESENTATION}

As alluded to previously, data representation is a major API issue. We identify three independent components of data representation. When each component is recognized explicitly, we can accommodate systematically the different representations adopted by all the packages. We first review the mathematical background before elborating on the representations.

The proposed API focuses on one mathematical transform only the Discrete Fourier Transform DFT. The natural domain for a onedimensional DFT is the space of complex periodic sequences:

$$
\left\{w_{j}\right\}, \quad w_{j} \in \mathrm{C}, \quad j=0, \pm 1, \pm 2, \ldots,
$$

and that there is a positive integer $n$ such that

$$
w_{j}=w_{n+j} \quad \text { for all } j .
$$

For general $d$-dimensional DFT, the natural domain is the $d$-dimensional periodic sequences

$$
\left\{w_{j_{1}, j_{2}, \ldots, j_{d}}\right\}, \quad w_{j_{1}, j_{2}, \ldots, j_{d}} \in \mathrm{C}, \quad j_{1}, \ldots, j_{d}=0, \pm 1, \pm 2, \ldots,
$$

and that there are positive integers $n_{1}, n_{2}, \ldots, n_{d}$ such that

$$
w_{j_{1}, j_{2}, \ldots, j_{d}}=w_{n_{1}+j_{1}, n_{2}+j_{2}, \ldots, n_{d}+j_{d}} \quad \text { for all } j_{i} \text {. }
$$

Our API considers the following (scaled version) transform:

$$
z_{k_{1}, k_{2}, \ldots, k_{d}}=\sigma \times \sum_{j_{d}=0}^{n_{d}-1} \cdots \sum_{j_{2}=0}^{n_{2}-1} \sum_{j_{1}=0}^{n_{1}-1} w_{j_{1}, j_{2}, \ldots, j_{d}} \exp \left(\delta 2 \pi \sqrt{-1} \sum_{i=1}^{d} j_{i} k_{i} / n_{i}\right)
$$

for $k_{i}=0, \pm 1, \pm 2, \ldots$, where $\sigma$ is an arbitrary real-valued scale factor and $\delta$ is either +1 or -1 . There is a well-known disagreement in whether to define forward transform to be the case $\delta=-1$ or the case $\delta=+1$. We will adopt the former and define the case $\delta=+1$ as the reverse transform.

It can be easily seen that the result of the transform is also complexvalued periodic with the same period as the input data. There are many common situations in which the input data are more special than just being complex periodic, such as real periodic for example. Special input domains in general lead to special output domains as well. Moreover, different representations are possible for each domain. These complications is a major reason why existing software have quite incompatible interfaces. We address the issues of different domains and representations next. 
Table 1 Domain Correspondence of Forward and Reverse DFT

\begin{tabular}{lr}
\hline Input & Output \\
\hline General & General \\
Real & Conjugate Even \\
Conjugate Even & Real \\
\hline
\end{tabular}

3.2.1 Domains. We consider three domains explicitly:

1 General Sequences: This is the largest domain, namely the domain of complex-valued periodic sequence:

$$
\left\{w_{j_{1}, j_{2}, \ldots, j_{d}}\right\}, \quad w_{j_{1}, j_{2}, \ldots, j_{d}} \in \mathrm{C}, \quad \text { and } \quad w_{\mathbf{j}+\mathbf{n}}=w_{\mathbf{j}} .
$$

2 Real Sequences: This is the domain of real-valued periodic sequences:

$$
\left\{w_{j_{1}, j_{2}, \ldots, j_{d}}\right\}, \quad w_{j_{1}, j_{2}, \ldots, j_{d}} \in \mathbf{R}, \quad \text { and } \quad w_{\mathbf{j}+\mathbf{n}}=w_{\mathbf{j}} .
$$

3 Conjugate-Even Sequences: An even sequence (analogous to an even function) is one that is symmetric at the origin: $w_{-\mathbf{j}}=w_{\mathbf{j}}$. A conjugate-even sequence is one that is even up to conjugacy:

$$
\left\{w_{j_{1}, j_{2}, \ldots, j_{d}}\right\}, \quad w_{j_{1}, j_{2}, \ldots, j_{d}} \in \mathrm{C}, \quad \text { and } \quad w_{\mathbf{j}+\mathbf{n}}=w_{\mathbf{j}}
$$

and

$$
w_{-j_{1},-j_{2}, \ldots,-j_{d}}=\bar{w}_{j_{1}, j_{2}, \ldots, j_{d}} .
$$

We do not consider more restrictive subdomains such as real-valued conjugate-even sequences, for example. The result of either forward or reverse transforms applied to data in one of the above subdomains yield result in another subdomain (see for example [1, 2]) as tabulated in Table 1.

We now consider the representation of each of the three domains. There are in fact two components of representation. First, the domains of periodic (infinite length) sequences are mapped bijectively to a space of finite-length sequences. Second, these finite-length sequences are packed into finite-length sequence of a built-in (native) data type. For example, a one-dimensional real-valued period- $n$ sequence $w_{j}, j=0, \pm 1, \pm 2, \ldots$, is naturally mapped into an length- $n$ sequence $x_{0}, x_{1}, \ldots, x_{n-1}$ where $x_{j}=w_{j}$ for $0 \leq j<n$. This finite-length sequence $x$, in some situations have to be packed into a array $X$ of type complex where

$$
\operatorname{Re}(X(j))=w_{2 j}, \quad \text { and } \operatorname{Im}(X(j))=w_{2 j+1} .
$$


The distinction of the two components of representation may seem redundant, but is in fact necessary if we are to accommodate lucidly the varieties of current practices such as in-place routines (that is, output overwritting input) where source and result domains are different (for example, the so called real-to-complex transforms). Moreover, explicitly recognizing these two components leaves flexibility for additional representations to be supported in the future.

\subsubsection{Representation - Mapping to Finite Sequences.}

For each domain, we support mappings to finite real-valued or complexvalued (or both) sequences. The sequences below are denoted by

$$
w_{j_{1}, j_{2}, \ldots, j_{d}}
$$

with $d$ positive periods

$$
n_{1}, n_{2}, \ldots, n_{d}>0
$$

1 General Sequences. A general sequence is mapped naturally

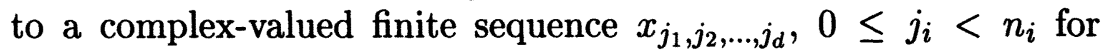
$1 \leq i \leq d$, where

$$
x_{j_{1}, j_{2}, \ldots, j_{d}}=w_{j_{1}, j_{2}, \ldots, j_{d}} \text {. }
$$

We also support a mapping of $w$ to two real-valued finite sequences $x_{j_{1}, j_{2}, \ldots, j_{d}}$ and $y_{j_{1}, j_{2}, \ldots, j_{d}}, 0 \leq j_{i}<n_{i}$ for $1 \leq i \leq d$, where

$$
x_{j_{1}, j_{2}, \ldots, j_{d}}=\operatorname{Re}\left(w_{j_{1}, j_{2}, \ldots, j_{d}}\right), \text { and } y_{j_{1}, j_{2}, \ldots, j_{d}}=\operatorname{Im}\left(w_{j_{1}, j_{2}, \ldots, j_{d}}\right)
$$

2 Real Sequences. A real sequence is mapped naturally to a realvalued finite sequence $x_{j_{1}, j_{2}, \ldots, j_{d}}, 0 \leq j_{i}<n_{i}$ for $1 \leq i \leq d$, where

$$
x_{j_{1}, j_{2}, \ldots, j_{d}}=w_{j_{1}, j_{2}, \ldots, j_{d}} .
$$

This is the only mapping we support for this domain.

3 Conjugate-Even Sequences. A conjugate-even sequence can be mapped into a complex-valued sequence $x_{j_{1}, j_{2}, \ldots, j_{d}}, 0 \leq j_{1} \leq$ $\left\lfloor n_{1} / 2\right\rfloor$, and $0 \leq j_{i}<n_{i}$ for $2 \leq i \leq d$, where

$$
x_{j_{1}, j_{2}, \ldots, j_{d}}=w_{j_{1}, j_{2}, \ldots, j_{d}} .
$$

This mapping is bijective because the complete periodic sequence can be reconstructed easily. Consider indices $j_{i}, 1 \leq i \leq d$ where $\left\lfloor n_{1} / 2\right\rfloor<j_{1}<n_{1}$ and $0 \leq j_{i}<n_{i}$ otherwise. We have

$$
w_{j_{1}, j_{2}, \ldots, j_{d}}=\bar{w}_{n_{1}-j_{1}, k_{2} n_{2}-j_{2}, \ldots, k_{d} n_{d}-j_{d}}
$$


by periodicity and conjugate eveness. It is easy to see that

$$
0<n_{1}-j_{1} \leq\left\lfloor n_{1} / 2\right\rfloor \text {, and } 0 \leq k_{i} n_{i}-j_{i}<n_{i}, \quad 2 \leq i \leq d .
$$

by chosing $k_{i}$ to be 1 when $j_{i}>0$ and $k_{i}$ to be 0 otherwise. Thus,

$$
w_{j_{1}, j_{2}, \ldots, j_{d}}=\bar{x}_{n_{1}-j_{1}, k_{2} n_{2}-j_{2}, \ldots, k_{d} n_{d}-j_{d}} .
$$

When the dimension of the sequence is one, we support a mapping of $w$ into a real-valued sequence $x_{j_{1}}, 0 \leq j_{1}<n_{1}$, where

$$
\begin{aligned}
x_{j_{1}} & =\operatorname{Re}\left(w_{j_{1}}\right), \quad j_{1}=0,1, \ldots,\left\lfloor n_{1} / 2\right\rfloor, \\
x_{n_{1}-j_{1}} & =\operatorname{Im}\left(w_{j_{1}}\right), \quad j_{1}=1,2, \ldots,\left\lfloor\left(n_{1}-1\right) / 2\right\rfloor .
\end{aligned}
$$

This mapping is also bijective. $w_{0}$ is always real-valued for a conjugate-even sequence. When $n_{1}$ is odd, $\left\lfloor\left(n_{1}-1\right) / 2\right\rfloor=\left\lfloor n_{1} / 2\right\rfloor$. Thus, the $x$ vector gives us $w_{j_{1}}$ for $0 \leq j_{1} \leq\left\lfloor\left(n_{1} / 2\right)\right\rfloor$. When $n_{1}$ is even, simple symmetry shows that $w_{n_{1} / 2}$ is real-valued as well. Thus, once again, the $x$ vector contains $w_{j_{1}}$ for $0 \leq j_{1} \leq\left\lfloor n_{1} / 2\right\rfloor$. Consequently, the $x$ sequence always yield $w_{j_{1}}, 0 \leq j_{1}<\left\lfloor n_{1} / 2\right\rfloor$, from which the whole $w$ sequence can be reconstructed by virtue of periodicity and conjugate eveness as discussed previously.

\subsubsection{Representation - Packing into Data Types. We} consider packing $\left\{x_{j_{1}, j_{2}, \ldots, j_{d}}\right\}, 0 \leq j_{i}<J_{i}, 1 \leq i \leq d$, a real-valued or complex-valued multi-dimension sequence, into real-valued or complexvalued multi-dimension sequence $\left\{X_{j_{1}, j_{2}, \ldots, j_{d}}\right\}$ of data items in real or complex data type. Note that the $J_{i}$ 's are not necessarily the periods of the sequence in question. The packing is a striaghtforward one-toone correspondence in the case of real packed into real or complex into complex:

$$
X_{j_{1}, j_{2}, \ldots, j_{d}}=x_{j_{1}, j_{2}, \ldots, j_{d}}
$$

The two other cases are as follows.

1 Real Packed into Complex. A real-valued sequence

$$
x_{j_{1}, j_{2}, \ldots, j_{d}}, \quad 0 \leq j_{i}<J_{i}
$$

is packed into data items of complex data type $X_{j_{1}, j_{2}, \ldots, j_{d}}, 0 \leq j_{1}<$ $\left\lceil J_{1} / 2\right\rceil$, and $0 \leq j_{i}<J_{i}, 2 \leq i \leq d$, by

$$
\operatorname{Re}\left(X_{j_{1}, j_{2}, \ldots, j_{d}}\right)=x_{2 j_{1}, j_{2}, \ldots, j_{d}} \text {, and } \operatorname{Im}\left(X_{j_{1}, j_{2}, \ldots, j_{d}}\right)=x_{2 j_{1}+1, j_{2}, \ldots, j_{d}} \text {. }
$$

When $J_{1}$ is odd, we further require that $\operatorname{Im}\left(X_{\left\lceil J_{1} / 2\right\rceil, j_{2}, j_{3}, \ldots, j_{d}}\right)=0$ for $0 \leq j_{i}<J_{i}, 2 \leq i \leq d$. 
Table 2 Representation and Defaults for All Domains

\begin{tabular}{ll}
\hline Domain & Representation \\
\hline General & $\begin{array}{l}\text { Complex into Complex (Default) } \\
\text { Complex into Real } \\
\text { Real into Complex } \\
\text { Real into Real }\end{array}$ \\
& $\begin{array}{l}\text { Real into Real (Default) } \\
\text { Real into Complex }\end{array}$ \\
Real & $\begin{array}{l}\text { Complex into Complex } \\
\text { Complex into Real (Default) } \\
\text { Real into Complex (for one-dimension only) } \\
\text { Conjugate Even }\end{array}$ \\
& Real into Real (for one-dimension only) \\
\hline
\end{tabular}

2 Complex Packed into Real. A complex-valued sequence

$$
x_{j_{1}, j_{2}, \ldots, j_{d}}, \quad 0 \leq j_{i}<J_{i}
$$

is packed into data items of real data type $X_{j_{1}, j_{2}, \ldots, j_{d}}, 0 \leq j_{1}<2 J_{1}$, and $0 \leq j_{i}<J_{i}, 2 \leq i \leq d$, by

$$
X_{2 j_{1}, j_{2}, \ldots, j_{d}}=\operatorname{Re}\left(x_{j_{1}, j_{2}, \ldots, j_{d}}\right), \text { and } X_{2 j_{1}+1, j_{2}, \ldots, j_{d}}=\operatorname{Im}\left(x_{j_{1}, j_{2}, \ldots, j_{d}}\right) \text {. }
$$

3.2.4 Options in Representation. The two mapping choices coupled with the two packing choices result in a maximum of four possible representations for some domains. These four representations are complex into complex (that is, mapped to complex, and then packed into complex), complex into real, real into complex, and real into real. For each domain, we pick as default one representation that is either most natural or most commonly adopted. Table 2 tabulates the options offered and the default.

3.2.5 Stride - Data Structure. After a periodic sequence of a certain domain is represented by a finite-sequence of data items of a specific data type, these data items reside in an array. All arrays in our interface are considered flat. Generically, we consider the distribution of a finite sequence

$$
X_{j_{1}, j_{2}, \ldots, j_{d}} ; \quad 0 \leq j_{i}<J_{i}, \quad 1 \leq i \leq d
$$


to a one-dimensional (flat) array $X$ of the same type. In general, we will be dealing with multiple transforms. That is, there are $M$ such sequences

$$
X_{j_{1}, j_{2}, \ldots, j_{d}}^{(m)} ; \quad 0 \leq j_{i}<J_{i}, \quad 1 \leq i \leq d, \quad 0 \leq m<M .
$$

The distribution we adopt of these multiple sequences is that of uniform stride, which is specified completely by distances of neighboring elements within each dimension or same elements belonging to neighboring sequences. Specifically,

$$
X\left(\operatorname{index}\left(I_{0} ; L_{1}, L_{2}, \ldots, L_{d}, L_{d+1} ; j_{1}, j_{2}, \ldots, j_{d}, m\right)\right)=X_{j_{1}, j_{2}, \ldots, j_{d}}^{(m)}
$$

where

$\operatorname{index}\left(I_{0} ; L_{1}, L_{2}, \ldots, L_{d}, L_{d+1} ; j_{1}, j_{2}, \ldots, j_{d}, m\right)=I_{0}+\sum_{i=1}^{d} j_{i} L_{i}+m L_{d+1}$.

The value $I_{0}$ is index of first element of $X$ (that is default to be 1 in Fortran for array defined without an explicit beginning index) and we call the parameters $L_{i}$ 's generic strides.

In the case of a single one-dimensional sequence, $L_{1}$ is simply the stride. The default setting of strides in the general muti-dimensional situation corresponds to the case where the sequences are distributed tightly into the array:

$$
L_{1}=1, L_{2}=J_{1}, L_{3}=J_{1} J_{2}, \ldots, L_{d}=\Pi_{i=1}^{d-1} J_{i}, L_{d+1}=\Pi_{i=1}^{d} J_{i} .
$$

When the user needs to change this default, $d+1$ values must be supplied as an integer vector to the routine that changes the stride options. In case of a single transform, the value $L_{d+1}$ can be arbitrary, but a valid integer nevertheless.

This explicit specification of strides offers much flexibility in representation of transposes and provide a straightforward correspondence to $\mathrm{C} / \mathrm{C}++$. Moreover, despite many advancements in Fortran compilers, explicit striding can still offer potential performance gain over the use of advanced language features in references to less common situations such as sections of arrays.

\subsection{ILLUSTRATIONS}

As an illustration, the case of representing complex input data (general domain) by two real vectors containing the real and imaginary part is the "Real into Real" representation denoted by the parameter 
DFTP_Param_Rep_RR. A code fragment to effect this (single precision) inplace transform is

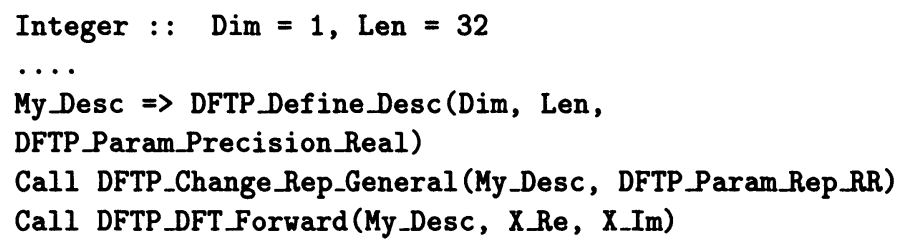

As another illustration, consider the case of representing real input data (real domain) in the default "Real into Real" and conjugate even data in the default "Complex into Real" representations. These default representations are natural for the default in-place transforms. For an input of $N$ elements stored contigously, the input array must have length $N+2$ to accommodate the output data. This situation is common. A code fragment to effect this in-place transform is

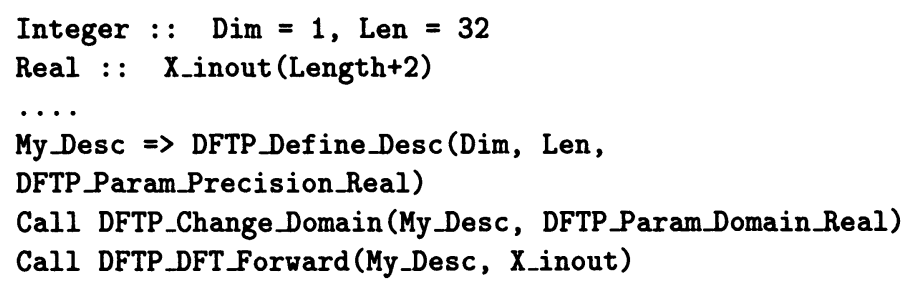

\subsection{OPTIONS SUPPORTED}

We now list the various options supported. Different subsets of the following list of options are supported by various software packages surveyed here.

- A scale factor for the transform. Default is 1 .

- Multiple number of transforms. Default is 1.

- Placement of result. Default is in place.

- Domain of input (general, real, or conjugate even). Output domain is implied. Default is general.

- Representations for domain. Default defined for each domain.

- Workspace requirement. Default is to make use of workspace.

In addition, we also propose two "advanced" options not commonly found.

- Allowance for reordering. This is an option that allows for the order of input or output to be scrambled (e.g. by a bit-reversal permutation). Default is no scrambling allowed. 
It is well known that the a number of FFT algorithms apply an explicit permutation stage that is time consuming [3]. Doing away with this step tantamounts to applying DFT to input whose order is scrambled or resulting in scrambling the order of the DFT result. In applications such as convolution and power spectrum calculation, the order of result or data is unimportant and thus permission of scrambled order is attractive if it leads to higher performance. Our API allows the following four options:

1 Input ordered, output ordered. This is the default.

2 Input ordered, output scrambled.

3 Input scrambled, output ordered.

4 Input scrambled, output scrambled. (Computation equivalent to that of case 1.)

All scramblings involved are digit reversal. Precisely, a length $J$ is factored into $K$ ordered factors $D_{1}, D_{2}, \ldots, D_{K}$. Any index $i$, $0 \leq i<n$, can be expressed uniquely as $K$ digits $i_{1}, i_{2}, \ldots, i_{K}$ where $0 \leq i_{l}<D_{l}$ and

$$
i=i_{1}+i_{2} D_{1}+i_{3} D_{1} D_{2}+\cdots+i_{K} D_{1} D_{2} \cdots D_{K-1} .
$$

A digit reversal permutation $\operatorname{scram}(i)$ is given by

$\operatorname{scram}(i)=i_{K}+i_{K-1} D_{K}+i_{K-2} D_{K} D_{K-1}+\cdots+i_{1} D_{K} D_{K-1} \cdots D_{2}$.

Factoring $J$ into one factor $J$ leads to no scrambling at all, that is, $\operatorname{scram}(i)=i$. Note that the factoring needs not correspond exactly to the number of "butterfly" stages to be carried out. In fact, the computation routine in its initialization stage will decide if indeed a srcambled order in some or all of the dimensions would lead to performance gain. The digits of the digit reversal are recorded and stored in the descriptor. These digits can be obtained by calling a corresponding inquiry routine that returns a pointer to an integer array. The first element is $K^{(1)}$ which is the number of digits for the first dimension, followed by $K^{(1)}$ values of the corresponding digits. If dimension is higher than one, the next integer value is $K^{(2)}$, etc.

We comment that simple permutation such as mod- $p$ sort [3] is a special case of digit reversal. Hence this option could be useful to high-performance implementation of one-dimensional DFT via a "six-step" or "four-step" framework [3]. 
- Allowance for transposition. This is an option that allows for the result of an high-dimensional transform to be presented in a transposed manner. Default is no transposition allowed.

Similar to that of scrambled order, sometimes in higher dimension transform, performance can be gained if the result is delivered in a transposed manner. Our API offers an option that allows the output be returned in a transposed form if performance gain is expected. Since the generic stride specification is naturally suited for representation of transposition, this option allows the strides for the output to be possibly different from those originally specified by the user. Consider an example where a two-dimensional result $y_{j_{1}, j_{2}}, 0 \leq j_{i}<n_{i}$, is expected. Originally the user specified that the result be distributed in the (flat) array $Y$ in with generic strides $L_{1}=1$ and $L_{2}=n_{1}$. With the option that allows for transposition, the computation may actually return the result into $Y$ with stride $L_{1}=n_{2}$ and $L_{2}=1$. These strides are obtainable from an appropriate inquiry function. Note also that in dimension 3 and above, transposition means an arbitrary permutation of the dimension.

\subsection{NAMING ORGANIZATION AND CONVENTIONS}

The design of the API leads naturally to four categories of routines:

1 Definition routine. This defines a transform by its dimension, periods, and precision required. There is only one routine, and the result is a data structure called descriptor that holds all the information and default settings.

2 Option changing routines. Since all options have a default value, setting a different choice amounts to a change. Each of the options listed previously has associated with it an option changing routine whose effect is to update the descriptor.

3 Computation routines. Since all the settings made are recorded in the descriptor, there is no need for different routine names for computation purposes. The only computation routines are for forward transform and reverse transform.

4 Inquiry routines. These routines allow retrieval of specific option setting as well as error conditions and side effects such as the actual scrambling performed should it be allowed. 
The naming conventions is DFTP_category_otherinfo. For example, the definition routine is DFTP_Define_Desc, that is, defining a descriptor. All the option changing and inquiry routines have the form

DFTP_Change_options, and DFTP_Inquire_options

for options such as Rep_Real and Stride_Input.

\section{API PROPOSAL}

The set of routines, derived types, and parameters are packaged in a Fortran 90 module named DFTPACK. Users must issue an "use" statement USE DFTPACK to make the routines and associated objects visible.

The module includes the following:

1 Derived Type: The module defines one derived type DFTP Desc which is the type for the DFT descriptor.

2 Parameters: The module defines a number of parameters that are used to specify settings of options.

3 Routines: The module provides four kinds of routines: definition routine, setting changing routines, computation routines, and inquiry routines.

\subsection{PARAMETERS}

The list of parameters are as follows:

1 Precision specification parameters:

- DFTP_Param_Precision_Real

- DFTP_Param_Precision_Double

2 Domain specification parameters:

- DFTP_Param_Domain_General

- DFTP_Param_Domain_Real

- DFTP_Param_Domain_ConEven

3 Domain representation parameters:

- DFTP_Param_Rep_CC (Mapped to complex, packed into complex.)

- DFTP_Param_Rep_CR (Mapped to complex, packed into real.)

- DFTP_Param_Rep_RC (Mapped to real, packed into complex.) 
- DFTP_Param_Rep_RR (Mapped to real, packed into real.)

4 Workspace specification parameters:

- DFTP_Param_Workspace_Allow (Use when deemed appropriate.)

- DFTP_Param_Workspace_Avoid (Avoid if possible.)

5 Ordering specification parameters:

- DFTP_Param_Ordering_00 (Ordered input, ordered output.)

- DFTP_Param_Ordering_OS (Ordered input, scrambled output.)

- DFTP_Param_Ordering_So (Scrambled input, ordered output.)

- DFTP_Param_Ordering_SS (Scrambled input, scrambled output.)

6 Transposition specification parameters:

- DFTP_Param_Transposition_Allow (Traspose result when appropriate.)

- DFTP_Param_Transposition_Avoid (Avoid if possible.)

\subsection{DEFINITION ROUTINE}

FUNCTION DFTP Define_Desc(Dimension, Periods, Precision) \& RESULT ( My_Desc)

TYPE (DFTP_Desc), POINTER :: My_Desc

INTEGER, INTENT(IN) : : Dimension, Precision

INTEGER, DIMENSION(*), INTENT(IN) : : Periods

Precision is specified by one of the precision specification parameter:

- DFTP_Param_Precision_Real, or

- DFTP Param_Precision_Double

\subsection{SETTING CHANGING ROUTINES}

\section{Scale Change}

The default scale is one. The routine name is overloaded.

SUBROUTINE DFTP_Change_Scale(My_Desc, New_Scale) TYPE (DFTP_Desc), INTENT (INOUT), POINTER :: MyDesc

REAL, INTENT(IN) :: New_Scale 
SUBROUTINE DFTP_Change_Scale(My_Desc, New_Scale)

TYPE (DFTP_Desc), INTENT (INOUT), POINTER :: My_Desc DOUBLE PRECISION, INTENT(IN) : : New_Scale

2 Number of Transforms

The default number of transforms is one.

SUBROUTINE DFTP_Change_NumTransforms (My_Desc, New_Number) TYPE (DFTP_Desc), INTENT (INOUT), POINTER :: My_Desc INTEGER, INTENT (IN) : : New_Number

3 Domain Change

The default domain is general sequence.

SUBROUTINE DFTP_Change_Domain(My_Desc, New_Domain)

TYPE (DFTP_DesC), INTENT (INOUT), POINTER :: My Desc INTEGER, INTENT(IN) :: New_Domain

New Domain is one of the domain specification parameters:

- DFTP_Param_Domain_General, or

- DFTP_Param_Domain_Real, or

- DFTP_Param_Domain_ConEven.

4 Representation Change for a Specific Domain

SUBROUTINE DFTP_Change_Rep_General (My_Desc, New_Rep)

SUBROUTINE DFTP_Change_Rep_Real (My_Desc, New_Rep)

SUBROUTINE DFTP_Change_Rep_ConEven (My_Desc, New_Rep)

TYPE (DFTP_Desc), INTENT (INOUT), POINTER :: My Desc

INTEGER, INTENT(IN) : : New_Rep

New Rep is one of the domain representation parameters:

- DFTP_Param_Rep_CC, or

- DFTP_Param_Rep_CR, or

- DFTP_Param_Rep_RC, or

- DFTP_Param_Rep_RR.

\section{Stride Change}

The default stride is that of tight distribution.

SUBROUTINE DFTP_Change_Stride_Input(My_Desc, New_Stride)

SUBROUTINE DFTP_Change_Stride_Output (My_Desc, New_Stride)

TYPE (DFTP_Desc), INTENT (INOUT), POINTER : : My_Desc

INTEGER, INTENT(IN), DIMENSION(*) :: New_Stride 
New_Stride is a vector specifying the generic strides (Section 3.2.5).

\section{Workspace Change}

The default is to allow the use of workspace.

SUBROUTINE DFTP_Change_Workspace(My_Desc, New_Workspace) TYPE (DFTP_Desc), INTENT (INOUT), POINTER : : My_Desc INTEGER, INTENT(IN) :: New_Workspace

New_Workspace is one of the workspace specification parameters:

- DFTP_Param_Workspace_Allow, or

- DFTP_Param_Workspace_Avoid.

\section{Ordering Change}

The default is that of ordered input and ordered output.

SUBROUTINE DFTP_Change_Ordering(My_Desc, New_Ordering) TYPE (DFTP_Desc), INTENT (INOUT), POINTER :: My_Desc INTEGER, INTENT(IN) : : New_Ordering

New_Ordering is one of the ordering specification parameters:

- DFTP_Param_Ordering_00, or

- DFTP_Param_Ordering_OS, or

- DFTP_Param_Ordering_SO, or

- DFTP_Param_Ordering_SS.

\section{Transposition Change}

The default is to avoid the output be transposed.

SUBROUTINE DFTP_Change_Transposition(My_Desc, New_Transposition) TYPE (DFTP_Desc), INTENT (INOUT), POINTER :: My_Desc INTEGER, INTENT(IN) : : New_Transposition

New_Transposition is one of the transposition specification parameters:

- DFTP_Param_Transposition_Allow, or

- DFTP_Param_Transposition_Avoid. 
Table 3 Attributes of two-argument computational routines

\begin{tabular}{lcclcc} 
Type & \multicolumn{1}{c}{ X } & Intent & Type & & Intent \\
\hline Complex & (double complex) & In & Complex & (double complex) & Out \\
Complex & (double complex) & In & Real & (double precision) & Out \\
Real & (double precision) & In & Complex & (double complex) & Out \\
Real & (double precision) & In & Real & (double precision ) & Out \\
Real & (double precision) & Inout & Real & (double precision) & Inout \\
\hline
\end{tabular}

\subsection{COMPUTATION ROUTINES}

The routine names are heavily overloaded. The number of parameters vary as well as the type and precision because of different domain and representation as well as the different situations between in-place and out-of-place interface. The first parameter is always the pointer to the descriptor whose intent is "inout." The data and result parameters are all declared as assumed-size rank-1 array DIMENSION $(0: *)$. The first form of the computation routine has two parameters following the descriptor. SUBROUTINE DFTP_DFT_\{Forward, Reverse $\}$ (My_Desc, X, Y) The parameters can have the following attributes:

The second form of the computation routines has four parameters following the descriptor. This is applicable to one case, namely, an out-of-place computation on transform on general domain where the representation is "RR."

SUBROUTINE DFTP_DFT_\{Forward, Reverse\} (My_Desc, X_re, X_im, Y_re, Y_im)

TYPE(DFTP_Desc), INTENT (INOUT), POINTER :: My_Desc

REAL, INTENT(IN), DIMENSION $(0: *):: \quad X_{\text {_re, }} X_{-i m}$

REAL, INTENT (OUT), DIMENSION $(0: *):: \quad Y_{\text {_re, }} Y_{\text {_im }}$

There is a corresponding routine for double precision as well. Finally, the thrid form of the computation routines has one parameter following the descriptor. These are all in-place routines.

SUBROUTINE DFTP_DFT_\{Forward, Reverse\} (My_Desc, X)

The parameters can have the following attributes:

\subsection{INQUIRY ROUTINES}

Finally, the module provides a set of inquiry routines in the form of function. One function is provided to return the error code. An error number of 0 implies normal completion. Meaning of non-zero error number can be implementation dependent. It is also possible that a 
Table 4 Attributes of inplace routines

Type

Intent

Complex (or double complex)

Inout

Real (or double precision)

Inout

Table 5 Description of inquiry routines

Function Name

DFTP_Inquire_ErrorNum
DFTP_Inquire_Dimension
DFTP_Inquire_Periods
DFTP_Inquire_Precision
DFTP_Inquire_NumTransforms
DFTP_Inquire_Domain
DFTP_Inquire_Rep_General
DFTP_Inquire_Rep_Real
DFTP_Inquire_Rep_ConEven
DFTP_Inquire_Stride_Input
DFTP_Inquire_Stride_Output
DFTP_Inquire_Workspace
DFTP_Inquire_Ordering
DFTP_Inquire_Digit_Reversal
DFTP_Inquire_Transposition

Return Value

Integer scalar
Integer scalar
pointer
scalar, DFTPACK parameters
Integer scalar
scalar, DFTPACK parameters
scalar, DFTPACK parameters
scalar, DFTPACK parameters
scalar, DFTPACK parameters
pointer
pointer
scalar, DFTPACK parameters
scalar, DFTPACK parameters
pointer
scalar, DFTPACK parameters

small set of error condition with associated non-zero error number can be made standard. Correspond to each option is an inquiry function that returns the current setting. The functions all take one input parameter which is the pointer to the current descriptor. On return, the function either returns an integer scalar, or a pointer to an integer array in the case where an array is needed for the return information.

\section{CONCLUSION}

We believe the proposed API achieves all the goals set out in Section 3. Our proposal is formulated in Fortran 90 together with a $\mathrm{C}$ binding that has an almost one-to-one correspondence with the Fortran counterpart. While the vast functionality supported requires a substaintial implementation effort, the proposed options can be supported incrementally. A reference implementation effort is underway. 


\section{References}

[1] E. Oran Brigham, The Fast Fourier Transform and Its Applications, Prentice Hall, New Jersey, 1988.

[2] Athanasios Papoulis, The Fourier Integral and its Applications, 2nd edition, McGraw-Hill, New York, 1984.

[3] Charles Van Loan, Computational Frameworks for the Fast Fourier Transform, SIAM, Philadelphia, 1992 


\section{DISCUSSION}

\section{Speaker: Ping Tak Peter Tang}

Robert van de Geijn : It seems to me that there really should be two descriptors. The first should describe the data, including length, storage method, and pointer to the actual data. The second should describe the operation to be performed. One benefit would be that one could take "views" of the data, which would clarify implementation.

Ping Tak Peter Tang : While further dividing the attributes of the problem and encapsulating them with more than one descriptor in principle allows for flexibility that may facilitate unforseen features that may be desired, the Discrete Fourier Transform is simple compared to, say, linear algebra, where many different operations (LU, SVD, Cholesky, etc.) might be applied to same data. Here we only have one operation, namely, the DFT. Doubtlessly, different algorithms will be used to effect the transform depending on the properties of the data, but it is best to hide that from the user.

Fred Gustavson : I applaud you for producing an API for the FFT. You have produced a very good design. Bernard Rudin, from IBM, and I suggested to Charles Van Loan the need for such an interface in 1995. Charles agreed. However, our effort did not materialize in an interface. Ping Tak Peter Tang: Thank you for the encouragement. I too feel strongly about a need for a common API. I will rely on colleagues like you to give me moral support and technical advice as I, and possibly other collaborators proceed on this project.

Anne Trefethen : Having a common API for DFT is important. Togeher with the BLAS, DFTs are functionality often provided by hardware vendors. At NAG, we interface to the vendor routines when available. How do we get the hardware vendors to buy into this common API?

Ping Tak Peter Tang : I plan to pursue two routes. First, a portable and public domain implementation of the API must be made available. This implementation must have a very reasonable performance compared to native implementations. Moreover, the software architecture must consist of components that vendors can easily replace with their existing routines with minimal effort. This will allow a vendor to take the reference implementation that supplies all the functionality and improve performance on the subset that the vendor has already optimized. Second, I intend to produce, and put into open source, Intel-specific implementations both for the 32-bit and 64-bit architectures.

Richard Hanson : The use of a descriptor hides certain initialization steps. These include computing the twiddle factors (complex multipli- 
ers). In threaded environments it seems that an initialization call to the descriptor logic will be necessary.

Ping Tak Peter Tang : I am just beginning on some implementation effort. I believe the API design is sound and will allow correct and thread-safe implementation without performance penalty. I agree that we must have a thread-safe implementation. 